\title{
GeoNotes: Social and Navigational Aspects of Location-Based Information Systems
}

\author{
Fredrik Espinoza, Per Persson, Anna Sandin, Hanna Nyström, Elenor Cacciatore \& \\ Markus Bylund \\ HUMLE Lab, Swedish Institute of Computer Science (SICS) Box 1263, 16429 Kista, \\ Sweden \\ \{espinoza, perp, sandin, hannan, elenor, bylund\}@sics.se
}

\begin{abstract}
Location-based information systems allow the user to access information in relation to the user's position in geographical space. This paper outlines navigational and social aspects of such systems. It is argued that location-based systems must allow users to participate as content providers in order to achieve a social and dynamic information space. Moreover, as these systems allow commercial and private users to annotate space with information on a mass-scale, information filtering techniques will become essential in order to prevent information overload and user disturbance. We present a number of content-based and social filtering techniques to support this. We discuss implications for implementation and we describe a system (GeoNotes), which takes some of these aspects into account.
\end{abstract}

Keywords. Real-world annotations, position-based services, social awareness, context awareness.

May 2001

SICS Technical Report T2001/08

ISSN 1100-3154

ISRN: SICS-T--2001/08-SE 


\section{Introduction}

Some location-based systems are centered on the notion of positioning people in relation to each other (e.g., [11]). Location-based information systems, on the other hand, position information. The basic idea is to connect pieces of digital information to a specific latitude-longitude coordinate via some mobile device, thereby 'attaching' them to a specific place in space. Later, users, again via some mobile client, can access that information. In this way, users will get the impression that the digital information is actually attached to a place in a way similar to post-its, graffiti and public signs and posters.

Quite a few systems have been working with this basic concept over the last five years within the fields of augmented reality, wearables and ubiquitous computing [8; $3 ; 1 ; 15 ; 19 ; 7,17,4]$. Some have used goggles as access medium [20]. Most, however, work with handheld devices, that may not provide the same stunning and spectacular visual effects, but still preserve the basic functionality.

It is our intention not to repeat the results of these projects, but rather to discuss the communicatory, social and navigational implications of the mass usage of a locationbased information system of this kind. We believe that the space of location-based information needs to be free for all users - not only professional content providers. Moreover, once we allow for mass-annotations, this will impose serious design challenges in terms of navigation and filtering. In the same way as the World Wide Web user eventually needed a wide range of navigation support (e.g. search engines, collaborative filters, news-groups, link lists, index views, navigation bars, and "webtips" in the press), so will users of location-based information systems.

First we discuss the communicatory and social functions of "traditional locationbased information systems', such as post-its, graffiti and posters. In the light of this, we then analyze some features and social shortcomings of digital location-based information systems, and present a more social emphasis for location-based information systems. Next, we address the design implications/requirements for such a social location-based information system in relation to the user's interaction with and experience of the system. Finally, we present an implementation - the GeoNotes system - that tries to address these design challenges.

Thus, the purpose of the paper is twofold. On a theoretical level, it seeks to analyze the social aspects of location-based information systems, and propose design solutions to support those aspects (both on a user-oriented and a technical level). On the other hand, we also present a system that goes some steps in the direction of the design we suggest.

\section{Social Functions of Post-its, Graffiti and Posters}

Although location-based information systems need not slavishly replicate the features of paper post-it notes, graffiti, signs and posters, such technologies may provide 
design inspirations for their digital counterpart. In particular, their communicatory and social functions may provide insights.

Although they do not adhere to all surfaces, post-it notes stick to almost everything in an indoor environment. Other than primitive sketches, multimedia content is not possible. However, they are dispensable, cheap and easily accessible. Since creator and reader share the same spatial context, the message can rely on short deictic expressions such as 'Make 10 copies of this!' without loss of understanding. Such referencing can be quite exact since the note can be placed on rather small object (e.g., pieces of paper, computer screens, mugs). Post-its are mostly used between peers and colleagues who know each other's habits, preferences, sense of humor and personality quite well. By exploiting and alluding to this shared context, messages need not be that long to contain much valuable information. Moreover, post-its need no greetings or wrapping up phrases as e-mails do.

At work, post-its are used to coordinate and plan tasks in order to make work more efficient. Reminding oneself and others is a central communicatory function of postits. In domestic environments they can have additional expressive and social bonding functions (e.g., 'Good morning! I love you very much!'). Such communication may be less 'serious' and task oriented, but may in fact constitute the most common usage of post-it technology.

While post-its are oftentimes directed to individuals and peers, graffiti - which can be both textual and graphical in nature - is mostly anonymous and intended for a mass-audience ('Stop the deforestation! Stop eating at McDonalds!'). Because of this, graffiti is annotating public rather than domestic places, for instance public restrooms, walls, buildings, and public transportation. Often it has a strong expressive function in terms of emotional reactions, social and ethnic identity, politics, prejudices, humor, and sex. Because of its subversive character, graffiti is particularly prohibited in places with strong commercial interests. Although most graffiti stands on its own, sometimes it spurs remarks and counterarguments from readers (cf. public note boards).

Signs and posters in public space are also oriented to a mass-audience. Most of them are commercial and attempt to stimulate consumption, whereas some are more informative in nature. Posters may be private, for instance 'This car for sale' or 'Apartment for rent in this area'. Note boards are information hubs where signs and posters cluster. People will make use of note boards to find and place information. Note boards are often situated in places where people pass through, e.g. entrances/exists of buildings and stores, bus stops, and waiting rooms.

\section{A More Social Location-Based Information System?}

For several reasons, digital location-based information systems within ubiquitous computing and context aware-applications have been focused on developing the technological aspects of attaching and accessing location-based information. While analog annotation technologies uphold key social and communicatory functions, these have often been overlooked in digital location-based information systems. 
For instance, with few exceptions, the information in those systems has been created by professional content providers, such as art institutions, museums, tourist organizations, and business interests $[8,3,1,15,19]$. Relying on professional content providers, these systems run the risk of making the information formal, official and impersonal. Although some users are great fans of museums and art galleries, these domains are not part of the fabric of everyday life, and they do not involve friends, families, (potential) acquaintances or other interpersonal relationships. Moreover, professionally created information often tends to be 'serious' and 'utility oriented' in ways in which post-its, graffiti and posters are not. The social, expressive, and subversive functions of analog annotation technologies tend to be overlooked.

Another problem with professional content is that information space easily becomes static. With large information spaces, maintaining and updating information is expensive and time consuming when relying on a few content providers. Since many users (and many information seeking situations) insist on information freshness, such an approach will eventually become untenable.

An alternative to this is to let information space free, allowing it to grow, expand and develop with users. Instead of making location-based information systems a vertical (one way) channel for information, by allowing and encouraging 'ordinary' users to provide, update, remove and comment information, the information space will become more of a horizontal communication medium between users. In this scenario, all users can leave traces in the system (and in the geography) for others to see, which will create a social awareness [10]. The information space overlaying physical space will become more social, reflecting the lives, concerns and social reality of the users in that space, rather than reflecting the views of some organization or authority.

Again, the analogy with the World Wide Web becomes relevant. It is precisely because no single authority controls the information that it has become such a success. Of course, the Web contains valuable 'professional' information, but it also allows all users to easily participate in creating, shaping and inhabiting the digital information space.

Some location-based information systems do allow users to create their own annotations or comments on the professional content, but few encourage it and make it the central aspect of the system. Marmasse \& Schmandt's [17] GPS enabled ComMotion system and Rekimoto's [20] goggle system focus on user's annotations, but these are mainly directed to oneself as reminders and documents. The social aspect is still lacking.

A system allowing any user to act as content provider will encounter new design challenges. If we allow users to annotate digital space en masse and without restrictions, we may achieve a socially rich environment, but information space will become cluttered with unstructured information. How will users be able to find relevant and timely information in a system that has no central information designer? How can the system (or user) know what annotations are important for a user when she is in a position which contains 5000 annotations? If we allow annotations to be 'pushed' to the user's mobile device, how can we allow the user to regulate the tradeoff between curiosity about the spatial annotations and the disturbance? How can we impose a structure on the information space, and what will this structure be like? 
No location-based information system has addressed these navigational issues. The Graffiti system [4], based on Wave LAN positioning and laptop computers as access terminals, enables users to annotate a university campus with virtual notes directed to friends and fellow students, but it provides little support for navigation.

The question is how to balance the trade-off between creating an open and social information space while still enabling people to find relevant information in that space. Below, we propose a number of techniques for navigational support for such a system. The underlying presumption in most of these techniques is that navigating digital or real-world information spaces is social in character. Computer Supported Collaborative Work (CSCW), collaborative filtering [14] and social navigation [18, 9] all emphasize that information seeking is not a single user activity, but often involves other people. We ask friends, authorities and experts for advice, or we study and learn from the ways in which they seek information. But we also study traces of others and follow their 'trails'. Many real world objects and places are able to aggregate usage, so called patina. A trail in the forest, for instance, will become greater if more people walk on it; a book in the library will be more worn if it is popular.

Navigation and work in digital information spaces may be enhanced by giving people access to the traces of other users or groups of users, who in some respect share similar interests with the navigator. Recommendation systems suggest books, movies and recipes, not by analyzing the content of the user profile and the content of the information item, but rather matching users' usage history in the system: 'people who bought the same books that you bought, also bought this one!' Clustering users into groups may be based on explicit voting as in Movielens ${ }^{l}$ or can be generated by machine learning techniques from the actions performed in the system [21]. Encouraging social awareness [10] and direct and indirect communication is a key objective for these researchers, not only because it supports work, but also because it enriches the experience of the system. For many user groups (e.g. young people) this can be seen as an end in itself.

In conclusion, our proposal for a location-based information system is social in two respects. On the one hand we allow all users to participate in creating the information space. On the other, we support navigation by collecting and aggregating users' usage of the system, and distribute this data to other users in some refined form.

\subsection{Interaction Requirements}

What are the implications for design of a more open and social location-based information system? In this section we discuss one design proposal in relation to interaction and user experience of the system. Some aspects of this proposal we implemented in our GeoNote system - which we will refer to throughout - whereas others are still awaiting implementation this year.

In relation to interaction, we identified three major design problems which all relate to navigation in one way or the other.

\footnotetext{
${ }^{1}$ http://movielens.umn.edu/
} 
- How can the system allow users to annotate geographical places by posting virtual notes on a mass-scale via their mobile devices? Specifically, the creator's and reader's understanding of the place/position of the annotation becomes crucial.

- What modes of accessing annotations are required in the system? How can we allow the user to browse, search or get a sense of the information at given place?

- How can filtering of information support users in finding relevant location-based annotations in this mass-scale information space?

Posting Notes. Posting an annotation - or a virtual note - should be easy and quick. Still the note should contain valuable information. Besides the information itself, which could be in textual or multimedia format, the sender needs to specify four components (see Fig. 1):

- A title. In the GeoNotes system the title will appear as a header in the list of notes in a particular place (see below and Fig. 2). In this way the user can get a sense of the content of the note by reading the title.

- The recipient to whom the note is directed. Although most notes will be open to any other user, the system must allow users to restrict access to the note, not only for privacy reasons, but also to support navigation. GeoNotes allows the user to configure lists of friends, which could be chosen as recipient. Alternatively, GeoNotes can be directed to individual friends or 'to oneself'.

- The signature of the creator is an important regulator of privacy. In some cases the identity and contact info to the creator is essential (e.g. for advertisements). In others, as with digital graffiti, the identity of the creator needs to be recognized but not identified (e.g. 'Kilroy'). In GeoNotes, any signature can be used when posting a note (e.g., 'SkateGirl', 'www.hem.passagen.se/anna', or 'Anna 070-633 15 07').

- A placement label of the creator's own choice. This 'tag' christens the place or object in which the note is intended to be posted. If the precision of the lat-long position coordinates is bad, either for the creator or for reader, such a label may help to focus the exact place for a note. By labeling the note, other users will be able to ascertain the correct placing without relying on the exact position. Such a semantic mechanism may be essential to understand the content of the note, and what it refers to in the spatial surroundings (e.g. deictic words such as this and here).

But even when the position precision is good, placement labels can be valuable ways of describing or discussing some aspect of a place. Even though the coordinates may be exactly the same, two users understanding of a place may differ. In fact, place refers less an objective coordinate, and more to a mental construct in the mind of the beholder. People may have very different understandings of the same place depending on preferences, personality, cultural dispositions, situation or goal. A label is a good way for the creator to indicate the intended aspect of any given place.

The matter of the placement label warrants special attention. We choose to use the labels approach in contrast to a stricter a priori approach such as [13]. Their location scopes are predefined tree-like structures that describe the real world as locations within locations. For example, 'Engineering' is a building within 'South Area', which in turn is an area within 'Campus'. When mapping a service or some other digital 
entity to the real world, one chooses the most appropriate location in such a structure. The system associates the entity with the specified location and to all of its 'superlocations', i.e. locations of which the specified location is a part. Thus, in our example, a GeoNote placed at 'Engineering' would also be found in 'South Area' and 'Campus'.

This method of organizing the world makes it possible to place entities completely unambiguously. However, it has some weaknesses: the structure must be designed in advance; it is based on an administrator's conception of the appropriate places (this is similar to [4] and [15]; it is coarse; it is static.

The label approach is superior on all counts: the structure is not preset but evolves during usage; the places are defined by users in response to the needs of the moment; the precision can be arbitrarily fine grained depending on what labels are defined; the structure is dynamic.

However, to make full use of the label approach one must implement label sharing. Label sharing means that labels for a given place are stored in the system and may be reused by other users. When a user creates a note, other notes in the vicinity (of that coordinate position) are scanned for their placement labels. This list of other users' labels is made available as a list of choices of possible placement labels. If the list is extensive, one may choose to sort the labels according to popularity. The creator may choose a label in the list or create a label of his or her own if none of them is appropriate.

Label sharing allow users to get a sense of how other users appreciate and interpret a given place, thus creating an awareness about the social connotations of a place (again emphasizing the indirect social aspect of information space). How such traces of others influence the creator's behavior and annotation activities is an interesting research problem that will have to be addressed in user studies. Another effect of label sharing is that valuable and efficient labels will be recycled more, whereas inefficient or extremely personal labels will be reused less. In this way, useful and popular place labels will continue to thrive in the system, similar to the ways in which vocabulary, expressions and grammar of natural language develops and transforms by its usage.

A label sharing system may be bootstrapped with predefined labels such as the places defined in previously mentioned systems or labels taken from the yellow pages or tourist databases.

The current version of GeoNotes implements posting of notes with title, recipient, signature and labels. It does not yet support label sharing. 


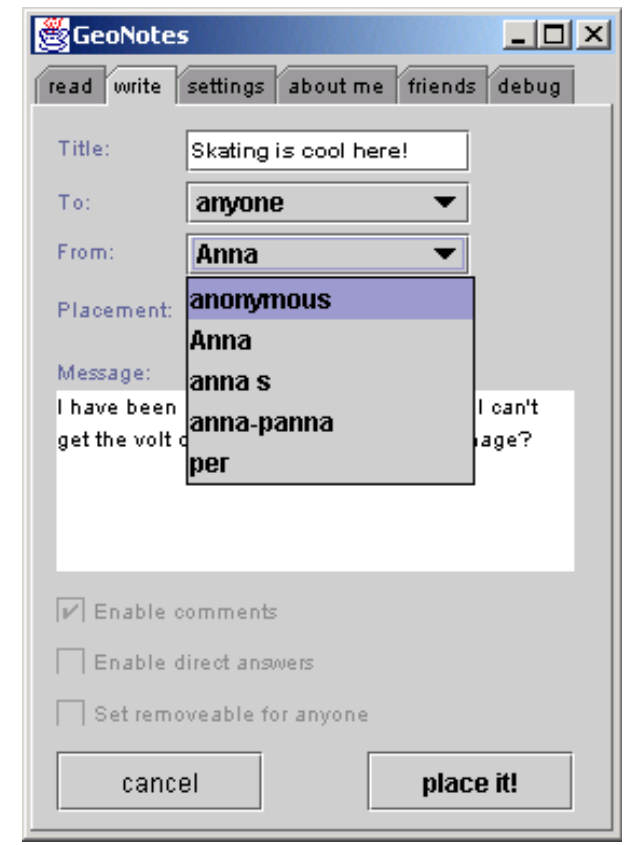

Fig. 1. Choosing a signature while posting a GeoNote

Accessing Notes: Pull and Push and In-Between. Users will want to access other people's notes in different ways, depending on preferences, situation and information need. In a retrieval mode, the user actively searches for or 'pulls' information, for instance by word-based search engines similar to the ones present on the World Wide Web today. In contrast to traditional web searches, location-based information systems may allow users to search for notes in confined geographical areas. Through some sort of map interface the user can indicate the area of interest and then execute the search. This is an interesting way in which searches can be constrained, but exactly how such searches differ from web-based ones and how they will be exploited by users are yet to be determined.

Of course, users will also be able to search for notes in the place/surroundings at which they are at the moment. If standing on the actual place, sharing the spatial context of the note creator may enable the information seeker to exploit this context in formulating more exact search queries. GeoNotes does not yet implement word-based search queries.

In a mixed push/pull access mode, the user is browsing the information space of notes but has no focused or well-defined information need. In contrast to active search, this mode of navigation is more explorative in nature, 'checking out' objects and information that might be of interest - cf. Benyon \& Höök's [2] distinction between way finding and exploration. The information seeker may, for instance, not be able to formulate her information needs in words, or she wants to learn more about a specific subject. In GeoNotes this mode of navigation is supported through the 'read' interface (see Fig. 2). On the left hand side of the screen is a pie chart that 
provides an at-a-glance overview of all GeoNotes at the current position (within a certain radius). The size of the circle could be relative to the total number of GeoNotes in order to give the user a sense of the GeoNote activity of the current position. The slices of the pie chart represent some automatically detected aspect of the GeoNotes. In our system, the categories are 'notes not visible (directed to others)', 'notes from anyone' and 'notes from friends', but one may consider other categories here, for instance sender oriented (private/commercial/governmental or regional/national/international). Such categorization gives the user a scent and flavor of the surrounding GeoNotes information space.

Pie chart circles for many locations within an area may also be placed on a grid map interface, with a pie chart within each grid. By allowing zooming and setting grid granularity, the user can, independent of the current location, get a sense of places with busy and poor GeoNotes activity. Although not yet implemented, such a feature would provide a social map, indicating the social 'patina' of geographical space.

On the right hand side of the screen are listed the titles of the individual GeoNotes for the current position (see Fig. 2). Clicking on an item in the list brings up the content of the individual GeoNote (see Fig. 3). In most cases the list will consist of quite a few notes, and there will be a need for sorting mechanisms, defining the order in which GeoNotes categories are placed in the list. Although one could imagine other valuable sorting mechanisms, the current version of GeoNotes implements sorting notes by 'popularity', 'recent', and 'notes directed to me'. By clicking the icons below the list (see Fig. 4), the category chosen will appear first in the list. Popularity measurement is based on reading, 'saving' and 'ignoring' any individual GeoNote (see Fig. 3 and discussion below). Popularity of a GeoNote increases when 'saved' and decreases when 'ignored', and the total popularity wight of a note is calculated using the formula: timesRead $+(2 *$ timesSaved $)-(2 *$ timesIgnored $)$. One could also imagine that the default ranking of notes in the list would be based on the filter mechanisms (discussed below).

In order to support 'on-the-move' navigation, GeoNotes will have the circle and list automatically transform as the user moves through space. While walking or riding a bus the circle will continuously re-segment and change size, representing the changing 'landscape' of GeoNotes. If the user finds something interesting, she can click on the circle at any time to freeze the transformation in order to explore GeoNotes at that place (even though the user continues to move away from it). 


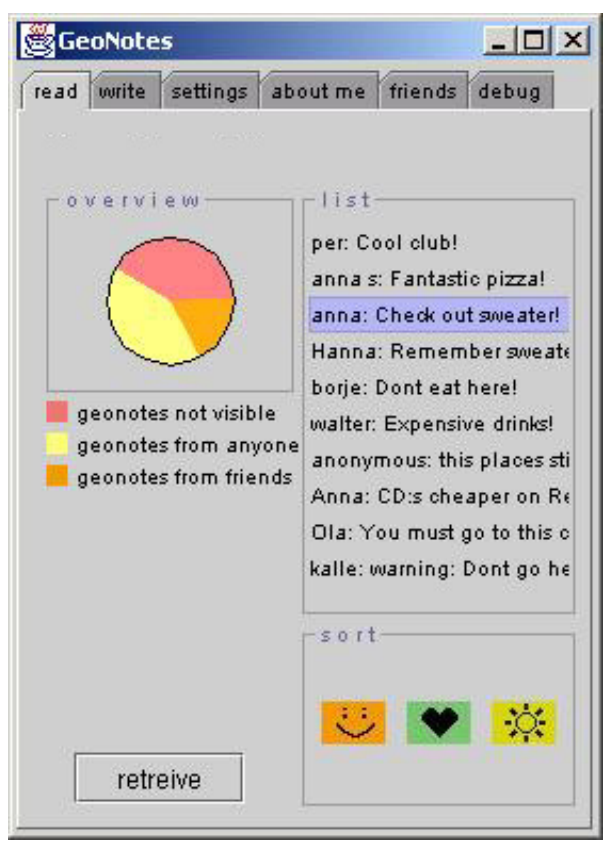

Fig. 2. The pie-chart circle provides an 'at-aglance' overview of the immediate GeoNote information space. On the right hand side of the screen is the list of the titles of individual GeoNotes

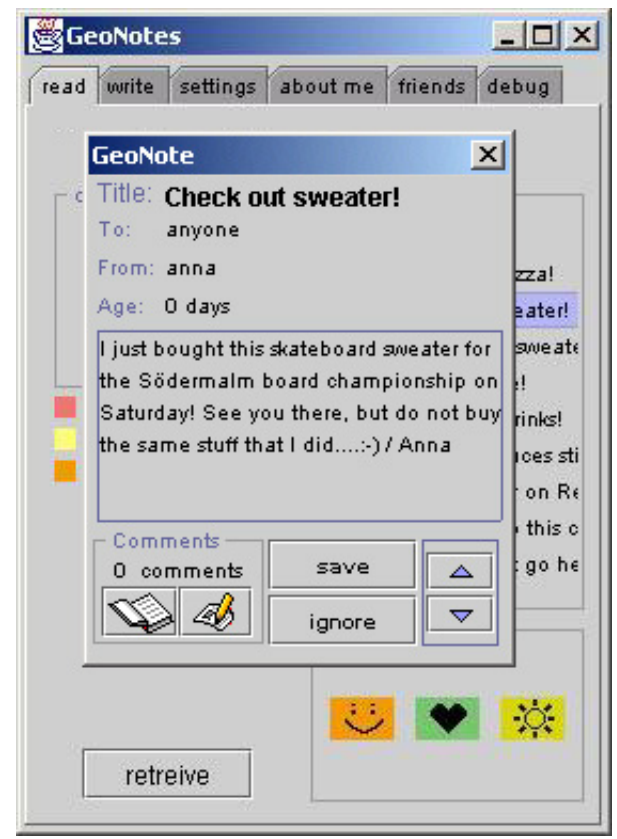

Fig. 3. Clicking in the list brings up the individual GeoNote

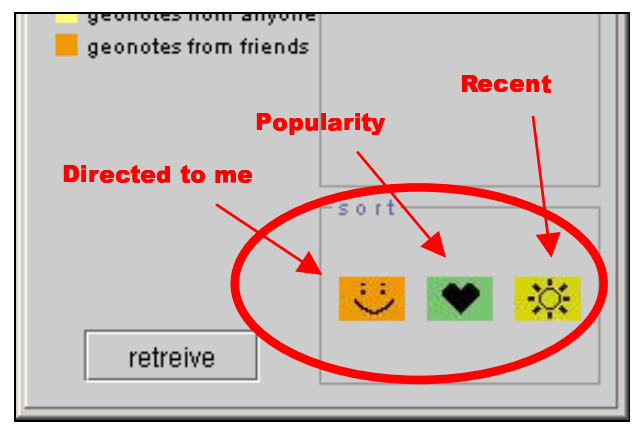

Fig. 4. The current GeoNotes application supports sorting by notes directed to me, most popular notes first, and the most recent notes first

The third way in which users will want to access notes is through notification. In a 'push' navigation mode, users will keep the mobile device in their pocket and when 
there is a high-ranking note in the vicinity, the device will signal and the individual note will pop up on the device. Most of the notes in a mass-scale annotation system will, however, not be relevant for the user in any given location. Many notes will for sure be commercial and non-commercial spam. The success of such a system fundamentally depends on its ability to filter out irrelevant notes. It is to those techniques we now turn.

Filtering notes. Notification systems alert, warn, remind or recommend users to consider some information or take some action. Successful notification systems must do this in a timely fashion, so that the reminders do not come too early or too late. Successful notification systems manage to evaluate the importance of the notification in relation to any given user, situation, task or goal at hand. Managing this trade-off between information value and disturbance is a formidable undertaking, which sometimes not even secretaries or coworkers succeed in [12].

Since work is often task-oriented and constantly shifting in terms of goals, notifications in such a setting will run the risk of being a disturbance rather than help (cf. Microsoft's paper clip). In a mobile setting, however, we can expect people to be more leisure oriented. On the metro, in bars, in schoolyards, with the family, on vacation and shopping on the town are places and situations in which there are fewer things that a user has to pay attention to and there are fewer deadlines. We can expect users to be more curious about the geographical and social environment, to be more willing to learn new things and to have a greater urge to be entertained. In one sense, notification systems and pushing of information onto the user may be more successful in leisure-oriented domains than in work related ones.

All the same, we must be careful when filtering pushed location-based information. We must make sure that the note is still informational or socially relevant for the user. As users move through space, the system must sort and rank notes in the vicinity, and only push those notes to the user that reach above a certain threshold. So what kind of filters may be efficient in this domain? Preferably, the filters should be based on both content and usage.

Content-based filters filter notes based on the user's interest and the content of the notes. In one such filtering technique, the user expresses his or her interest with a set of keywords of his or her own choice. Here one can imagine more or less sophisticated combinatorial and Boolean search methods. As a user moves through space, the system constantly scans available notes at any location and rates higher notes that match the user's keywords. This makes it more likely (or even certain, depending on the user's preferences) that some notes will be pushed to the user. As the interests of the user changes, he or she should be able to add or delete keywords. In GeoNotes, such a 'mobile search engine' is yet to be implemented.

In addition to this, the system may also collect usage data about how users read, click, save, choose, comment etc. notes in the system. Such actions can in different degrees be seen as voting for any given piece of information, which can then be used to rank notes. For instance, once the user has read a note, this note should probably be ranked lower next time the user enters the same location. The user must also be able to ignore the note, or even ignore the sender, which means that the note/any note from the sender will not be pushed to the user again (see Fig. 3). This function will be useful in situations with spam-GeoNotes, but probably requires that the GeoNote 
service stands fairly commercially independent. On the other hand, there should be mechanisms by which a user wants to 'save' valuable and reusable notes. Such a note will be given a high ranking next time the user is at the same location.

Usage based filters will become most forceful, however, when usage data is aggregated and matched with other users. Assuming that navigation is social, such data may be valuable in creating social filters, similar to those of collaborative filtering and social navigation. Simple cases of this are 'notes most recently put', 'notes most read' or 'notes most saved/commented'. Such filters or sorting mechanisms may be valuable in situations where freshness and popularity of the information is important.

In these cases, other users are anonymous and without identity, which may make the filters somewhat weak. The usefulness of following the traces of other people's behavior may be related to what kind of people that have left those traces. Trails may lead astray and the navigator wants to make sure that the person(s) who produced a trail was on the right track (in relation to my present information need). Thus, in order for collaborative filtering to work well, we may need to know the identity or some property (interest, hobbies, goals, age, nationality etc) of the other users in the system. At the same time, the system should be able to extract those aspects automatically without any major user overhead.

One simple way of doing so is to use the friends on the telephone list as a basis for ranking notes. 'Notes put/read/rejected or commented on by friends/a particular friend' are forceful filters since users will know the tastes and preferences of their peers and acquaintances. The navigator can position his or her own preferences in relation to theirs. The ways in which experts, authorities and celebrities have $\mathrm{put} / \mathrm{read} / \mathrm{rejected}$ and commented upon notes may also have relevance for a user. Moreover, occasionally rather basic aspects of other people might be valuable. When abroad, shared national identity may be a quite valuable feature. When making a tourist trip to Teheran, for instance, I might want to know about all notes posted by fellow Swedes (i.e. users that have registered a GeoNote service in Sweden).

If we allow the system to keep track of posting, reading, picking, rejecting and commenting on notes for each user, then we will also be able to automatically cluster unknown users in 'interest groups' and then base the ranking on this. This is the core feature of collaborative filtering techniques and recommendation systems $[14,21]$. The system, of course, does not 'know' the content of such interest groups, only that the users in the cluster have behaved in similar ways in the system. If someone in a user's cluster has put or read any notes in the vicinity that the user has not read, then this note would be ranked higher and potentially be pushed to the user. The ranking is thus based on automatic 'matching' of the usage history of users.

In a system that logs usage, it will also be possible to see all notes that were created by a specific friend, group of people or cluster of users (e.g. 'show me all notes Peter posted when he was traveling in Thailand last year'). One could also imagine a time slider, showing note-creating activities within any given geographical area over a period of time. Such a tool could be valuable for sociologists and historians in describing events and societal transformations.

Since the current version of GeoNote does not yet support logging of individual users' actions, only general and basic social filters are possible, for instance notes 'most read' and 'recently put'. 
Setting filter preferences may be the responsibility of the system, or may be left up to the user, depending on type of user. Minimally, however, there should be a simple mechanism by which the user can adjust the general 'push activity' of the system. Users' navigational modes may shift abruptly. In some situations the user will accept frequent note suggestions from the system; at other times, users will not want to be disturbed, or disturbed only when there is something really important (a top ranking note). Thus there should be an activity slider, which determines the general push activity of the system, independent of the content and usage-based filters. This is yet to be implemented in GeoNotes.

\section{Implementation}

Above, we described the interaction requirements for a real world annotation service with social filtering. We will now focus on our implementation of GeoNotes.

\subsection{Requirements}

The implementation of the GeoNotes service aims to meet a number of implementation requirements. These stem partly from the interaction requirements described above and partly from implementation constraints.

- The service must be able to store and retrieve data such as content, author, location, etc. about notes. Most of this data must be accessible and updateable by all users. Thus, the implementation should consist of at least two main parts: a client part that can execute locally on an end-user's mobile device and a server part that provides common functionality between all clients. The main reason for this is that the notes themselves and the meta-data about usage of the notes have to be available centrally to all users. Another reason relates to the fact that GeoNotes is a mobile service and as such it is subject to network connections of varying quality, and even complete loss of network connectivity. However, it is desirable to maintain some level of functionality when using the service off-line. The user might for example want to browse stored notes or edit user preferences. In cases of low bandwidth connections, the separation between client and server helps maintain a high level of quality of service since local functionality on the mobile device minimizes network traffic.

- User privacy must be maintained. Firstly, users should be able to remain anonymous while posting notes. The anonymous type of posting should complement posting under an alias and posting with a real name. Secondly, the set of personal data regarding which notes have been posted, which notes have been read, where the user posted notes, etc, must be protected from other users. Thirdly, it should be possible to address notes to specific persons or to the user group at large. This makes it possible to post notes that only certain users have access to.

We implemented the GeoNotes service in four parts as shown in the diagram in Fig. 5. 


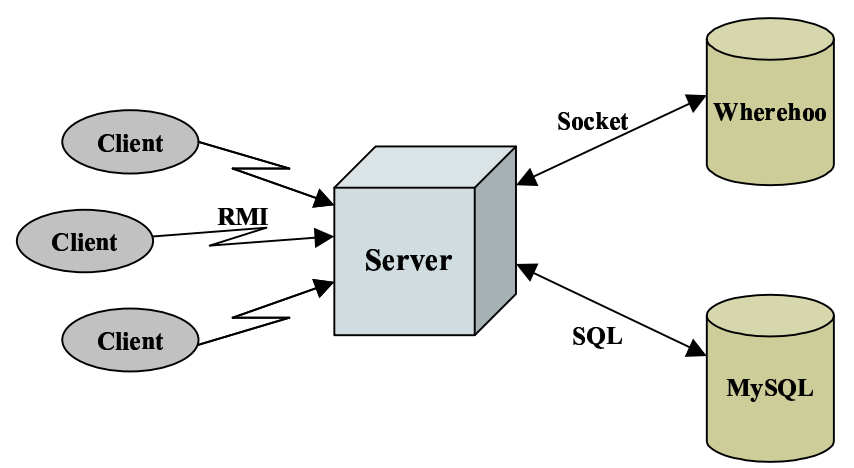

Fig. 5. The GeoNote System Architecture. Clients connect to the server using RMI. The server uses a socket connection to store content data about notes in the Wherehoo database and SQL commands to store meta data about the usage of notes in the MySQL database

\subsection{Client}

The client contains functionality that is specific to individual users. Such functionality includes authoring, reading, and sorting of notes. Personal service data (user name and personal information) and user client settings (aliases, lists of friends, etc.) are also stored with the client.

The client also provides a device dependent user interface to the functionality of the GeoNotes service. Only one such user interface has been implemented so far (a Java Swing based GUI), but the client is designed to allow for the implementation of other interfaces as well (e.g. based on HTML or WML). The Swing based user interface allows users to post notes, retrieve notes, and to manage user settings. We have also provided a simple debug panel that is useful for simulating the user's position if positioning technologies are unavailable.

The client implementation does not assume a particular positioning technology, but relies on the assumption that a latitude/longitude coordinate of its user is pushed to the client. The client can use any source of positioning information such as GPS or GSM if a plug-in Java class that represents it is installed. During the development of the service we built such a plug-in for a context simulator tool called QuakeSim [6]. QuakeSim is a graphical 3D tool that simulates real world space and outputs context information about actions that take place in the simulation. For GeoNotes, we used QuakeSim to simulate the movement and position of users in a 3D model of a wellknown area on Södermalm in Stockholm.

The implementation of the client allows the use of many different underlying protocols for communication between client and server. This is desirable because the client is intended for use on various mobile platforms that provide varying support for communication protocols. However, so far the use of RMI has been sufficient. Choosing to use RMI was straight forward because of its relative ease of use. Alternative protocols include custom use of HTTP or method invocation using CORBA or SOAP. 
We implemented the client as an sView service component [5]. sView is a platform for user access to electronic services. It is based on the concept of a service briefcase in which the user places and runs his or her services. The briefcase is able to move around the network to better service the user.

The usage of sView brings several advantages. Firstly, an sView service component is inherently mobile and persistent. Secondly, sView automatically packages functionality and data during migration or storage of the service component. Without sView, we would have had to implement all this functionality separately. In particular, persistence of personal user data and user client data is an important feature that is cumbersome to handle separately. Using sView, we can almost entirely leave such details to the underlying system.

We have also implemented a version of the client that runs as a standalone Java application. This version however, lacks the mobility and persistence features described above.

\subsection{Server}

The base server handles transactions of notes for all clients. It performs requests from clients to insert notes, retrieve notes from a location, and to update a single note.

It stores the actual note data (its contents), be it text, audio, or video, as an entry in the Wherehoo database [23]. Wherehoo is a service for storing location dependent data in a client independent manner. It stores data coupled to a field that describes the physical location of the data as a latitude/longitude position. To retrieve data, one specifies the location and a radius and the service returns all entries in that area.

The server enters individual GeoNotes into Wherehoo by sending an insert command with the note content data and the position as parameters. It retrieves GeoNotes by sending a request command with the position and the radius as parameters.

The server stores meta information about the usage of the note, such as the number of times it has been read, when it was last read, and so on, as data in the MySQL database. When a note is posted the server creates an entry in the MySQL database and the resulting note ID is then used to tag the note content data when it is inserted into Wherehoo.

By storing the content data in Wherehoo separately from the GeoNotes service specific meta data in the MySQL database, client types other than GeoNotes clients are able to access the content data. However, special care has to be taken to ensure that private notes are not publicly available. We currently have made no provisions for this but it is straightforward to add an extension to the server to use encryption on note data before inserting it into Wherehoo.

\section{Conclusions}

Mobility seems to set completely new constraints on information technology usage. Things that users would not want to do, not dare to do, or have no time to do at their stationary work terminals, people will want to do with their mobile devices when in 
bars, cafés, on public transportation, or school breaks. GeoNotes creates a social awareness that encourages play, expressiveness and personal identity formation. Such aspects tend to be central for large user groups, e.g. teenagers [16, 22].

The GeoNotes system tries to bridge quite disparate areas of research within the Human-Computer Interaction community. It tries to blur the boundary between physical and digital space (ubiquitous computing, augmented reality), and at the same time strives to socially enhance digital space (CSCW, collaborative filtering, social navigation). In combination, it seeks to socially enhance physical space by letting users leave virtual conscious and non-conscious traces that are tied to specific geographical positions. When these traces are available to other users, we will be able to enhance and enrich the social awareness that already exists in physical space. Users will be able to observe, create, and participate in large-scale social patterns in geographical space. The societal and sociological implications of such an information space in terms of public discourse, democracy, urban planning and public places, are yet to be determined.

\section{Acknowledgments}

Jarmo Laaksolahti, Peter Lönnqvist, Fredrik Olsson \& Martin Svensson took part in the initial GeoNotes brainstorming sessions. Kia Höök and Jussi Karlgren provided valuable inspiration and critique. Jim Youll helped with the Wherehoo related aspects of the server.

\section{References}

1. Abowd, G, Atkeson, C., Hong, J., Long, S., Kooper. R. \& Pinkerton, M. (1997) Cyberguide: A mobile context-aware tour guide, Wireless Networks, 3 (1997), 421-433.

2. Benyon, D. \& Höök. K. (1997) Navigation in information spaces: supporting the individual, In INTERACT'97, Sydney: Chapman \& Hall.

3. Broadbent, J., and Marti, P. (1997) Location Aware Mobile Interactive Guides: usability issues, in Proceedings of the Fourth International Conference on Hypermedia and Interactivity in Museums (ICHIM97).

4. Burrell, J. \& Gay, G. (2001) Collectively Defining Context in a Mobile, Networked Computing Environment, in Extended Abstracts, CHI'01, ACM Press.

5. Bylund, M., (2001) "sView - Personal Service Interaction", Ph.Lic. thesis, Computing Science Department, Uppsala University, Sweden

6. Bylund, M. \& Espinoza, F., (2001) "Using Quake III Arena to Simulate Sensors and Actuators when Evaluating and Testing Mobile Services", In Extended Abstracts, CHI 2001 Conference on Human Factors in Computing Systems, March31-April 5, 241-243

7. Caswell, D. \& Debaty, P. (2000) Creating Web Representations for Places, Proceedings of HUC 2000, Bristol, England, pp. 114-26.

8. Cheverst, K., Davies, N., Mitchell, K., Friday, A. and Efstratiou, C. (2000) Developing a context-aware electronic tourist guide: some issues and experiences; Proceedings of the CHI'OO, ACM Press, $17-24$. 
9. Dieberger, A., Dourish, P., Höök, K., Resnick, P., and Wexelblat, A. (2000) Social Navigation: Techniques for building more usable systems, in Interactions, NovemberDecember issue, ACM, 2000

10. Dourish, P. \& Bellotti, V. (1992) Awareness and coordination in shared workspaces. Proceedings of CSCW'92.

11. Holmquist, L-E, Falk, J. and Wigström J. (1999) Supporting Group Collaboration with Inter-Personal Awareness Devices, Journal of Personal Technologies, Special Issue on Hand-Held CSCW, Springer Verlag, 1999.

12. Hudson, S. \& Smith, I. (1996) Techniques for Addressing Fundamental Privacy and Disruption Tradeoffs in Awareness Support Systems, in Proceedings of CSCW'96, 24857.

13. José, R., \& Davies, N., (1999) Scalable and Flexible Location-Based Services for Ubiquitous Information Access, In Handheld and Ubiquitous Computing, First International Symposium, Karlsruhe, Germany, September 1999.

14. Konstan, J.A., Miller, B.N., Maltz, D., Herlocker, J.L., Gordon, L.R., Riedl, J. (1997) GroupLens: Applying collaborative filtering to Usenet news, Communications of the ACM 40 (3), 77-87.

15. Leonhardi, A., Kubach, U., Rothermel, K., Fritz, A. (1999) Virtual Information Towers A Metaphor for Intuitive, Location-Aware Information Access in a Mobile Environment, Proceedings of the Third International Symposium on Wearable Computers (ISWC'99), San Fransisco, CA, USA, IEEE Press, 1999.

16. Ling, R (1999) "We release them little by little": maturation and gender identity as seen in the use of mobile telephony, Telenor R\&D report 5/99, Norway.

17. Marmasse, N \& Schmandt, C. (2000) Location-Aware Information Delivery with ComMotion, Proceedings of HUC 2000, Bristol, England, pp. 157-171.

18. Munro, A., Höök, K. \& Benyon, D. (eds.) (1999) Social Navigation in Information Space, London: Springer.

19. Pascoe, J. (1997) The Stick-e Note Architecture: Extending the Interface Beyond the User, in Proceedings of IUI'97, 261-64.

20. Rekimoto, J., Ayatsuka, Y., (1998) Augment-able Reality: Situated Communication through Physical and Digital Spaces. In Proceedings of the $2^{\text {nd }}$ International Symposium on Wearable Computers, Oct 19-21, 1998, Pittsburgh, Pennsylvania, USA.

21. Svensson, M., Höök, K., Laaksolahti, J. \& Waern, A. (2001) Social Navigation of Food Recipes, Proceedings of CHI'01, ACM Press, 341-48.

22. Weilenmann, A. (2000) Negotiating Use: Making Sense of Mobile Technology, The Journal of Personal Technologies, no. 4, Springer Verlag.

23. Youll, J. \& Krikorian, R. (2000) Wherehoo Server: An interactive location service for software agents and intelligent systems, Workshop on Infrastructure for Smart Devices How to Make Ubiquity an Actuality, The Second International Symposium on Handheld and Ubiquitous Computing, Bristol (UK), September 27, 2000 